\title{
Consensus-based decentralized clustering for cooperative spectrum sensing in cognitive radio networks
}

\author{
WU QiHui ${ }^{\dagger *}$, DING GuoRu ${ }^{\dagger}$, WANG JinLong, LI XiaoQiang \& HUANG YuZhen \\ Institute of Communications Engineering, PLA University of Science and Technology, Nanjing 210007, China
}

Received November 1, 2011; accepted January 11, 2012; published online April 1, 2012

\begin{abstract}
A large number of previous works have demonstrated that cooperative spectrum sensing (CSS) among multiple users can greatly improve detection performance. However, when the number of secondary users (SUs; i.e., spectrum sensors) is large, the sensing overheads (e.g., time and energy consumption) will likely be intolerable if all SUs participate in CSS. In this paper, we proposed a fully decentralized CSS scheme based on recent advances in consensus theory and unsupervised learning technology. Relying only on iteratively information exchanges among one-hop neighbors, the SUs with potentially best detection performance form a cluster in an ad hoc manner. These SUs take charge of CSS according to an average consensus protocol and other SUs outside the cluster simply overhear the sensing outcomes. For comparison, we also provide a decentralized implementation of the existing centralized optimal soft combination (OSC) scheme. Numerical results show that the proposed scheme has detection performance comparable to that of the OSC scheme and outperforms the equal gain combination scheme and location-awareness scheme. Meanwhile, compared with the OSC scheme, the proposed scheme significantly reduces the sensing overheads and does not require a priori knowledge of the local received signal-to-noise ratio at each SU.
\end{abstract}

cognitive radio networks, spectrum sensing, decentralized clustering, unsupervised learning, consensus theory

Citation: Wu Q H, Ding G R, Wang J L, et al. Consensus-based decentralized clustering for cooperative spectrum sensing in cognitive radio networks. Chin Sci Bull, 2012, 57: 3677-3683, doi: 10.1007/s11434-012-5074-6

In cognitive radio networks (CRNs), secondary users (SUs) opportunistically exploit spectrum holes [1] left by primary users (PUs) to improve utilization of the wireless spectrum. To determine the temporal or/and spatial spectrum holes, a main challenge is reliable and efficient spectrum sensing. Compared with the single-SU spectrum sensing methods [2], cooperative spectrum sensing (CSS) among multiple SUs can be employed to tackle the problem of hidden primary receivers [3] and wireless channel fading (e.g., shadowing and multi-path fading) [4] by exploiting multi-user spatial diversity [5]. There have been recent comprehensive surveys on CSS in the literature [6,7].

In terms of whether a fusion center exists, previous works can be mainly classified as those on centralized CSS and those on decentralized CSS. A number of centralized

$\dagger$ These authors contributed equally to this work.

*Corresponding author (email: wqhqhw@163.com)
CSS techniques have been proposed, including methods of hard combination [8-10], soft combination [11,12] and quantized combination [13]. To further reduce sensing overheads (e.g., time and energy consumption) and improve the network scalability and robustness, there has been a recent focus on the design of decentralized CSS schemes. Decentralized CSS has been modeled as an evolutionary game in which each SU learns its best strategy (to collaborate or not) from strategic interactions with other SUs [14]. The network [14] is a single-hop network; i.e., each SU directly exchanges information with all other SUs. To balance the inherent tradeoff between the performance (i.e., detection probability) and the cost (i.e., the increment of false alarm probability) of cooperation, a decentralized CSS strategy has been developed from the theory of coalition formation game [15]. That scheme is not fully decentralized because of the existence of coalition heads. A fully decentralized CSS scheme has been proposed [16] on the basis of 
average consensus theory [17], where SUs iteratively exchange their sensing outcomes with one-hop neighbors before reaching an agreement (i.e., the average of their initial outcomes) on the presence of the PU. That scheme can be regarded as a decentralized implementation of the wellknown equal gain combination (EGC) scheme [12].

Although the problem of CSS has been extensively researched, there are still many interesting issues that need to be addressed. First, when the network is of large scale (i.e., both the number of SUs and the geographical space that they are distributed in are large), the sensing overhead of cooperation is likely to be huge if all SUs participate in cooperation. Moreover, the detection reliability of SUs at different locations is likely to vary significantly. Although there are related works such as those on sensor selection [18] or clustering-based CSS (e.g., [19] and references therein) that aim at tackling this issue, to the best knowledge of the authors, there is still no fully decentralized (i.e., no central coordinator) scheme.

In this paper, we design a decentralized clustering-based CSS scheme based on recent advances in unsupervised learning technology [20] and consensus theory [17]. Unsupervised learning is receiving much attention in the field of pattern recognition and machine learning [20]. The main idea is "learning without a teacher", which inherently has something in common with decentralized network operation. Consensus theory is initially bio-inspired (e.g., the flocking of birds and swarming of honeybees), and it plays a vital role in the field of distributed control and decision making, multi-agent cooperation and decentralized data fusion in wireless sensor networks (see [17] and references therein).

Compared with existing works, the proposed scheme has three distinct features:

(i) The network operation is fully decentralized. In the proposed scheme, no central coordinator (e.g., base station or access point) is needed and all information exchanges are among one-hop neighbors only. Decentralization has many advantages, such as robustness against an isolated point(s) of failure, higher scalability and reduced energy consumption.

(ii) The complexity of the proposed scheme is very low. On one hand, each SU does not need a priori knowledge of its local received SNR or location information of the PU and other SUs. On the basis of accumulative power received from the PU only, the SUs attempt to perform reliable CSS in an ad hoc manner. On the other hand, there is no need to spend extra time obtaining the observations used for decentralized clustering. In fact, the observations can be learned off-line by exploiting historical sensing results, which is explained in detail later.

(iii) The proposed scheme has great detection reliability with significantly reduced sensing overheads. To study the characteristics of large-scale CRNs, we adopt a practical wireless channel model consisting of path loss, log-normal shadowing, and multi-path Rayleigh fading. Abundant numerical simulations have been carried out to validate the advantages of the proposed scheme.

\section{System model and problem formulation}

\subsection{Network model and assumptions}

Consider a network consisting of one PU and $N$ SUs on the two-dimensional plane as in Figure 1. Without loss of generality, we assume that the PU is located at the center of an $L \times L$ square area and the $N$ SUs are uniformly distributed in the area. Note that the following proposed scheme can be further extended to the case of multiple PUs. The network is modeled as an undirected graph $G=(V, E)$ with vertex set $V$ and edge set $E$. The one-hop-connected relationship between SUs is represented by an adjacency matrix $\boldsymbol{A} \in R^{N \times N}$. If $\mathrm{SU} i$ and $\mathrm{SU} j$ are one-hop connected, then $\boldsymbol{A}_{i j}=\boldsymbol{A}_{j i}=1$; otherwise, $\boldsymbol{A}_{i j}=\boldsymbol{A}_{j i}=0$. SU $i$ can communicate with its one-hop neighbors $N_{i}:=\left\{j: j \in V, \boldsymbol{A}_{i j}=1\right\}$ only. The number of neighbors of SUi is referred to as the degree of $\mathrm{SU} i$, which is defined as $D_{i}:=\left|N_{i}\right|$. We further assume that no SU needs a priori location information of the PU and other SUs. On the basis of the power received from the PU only, the $N$ SUs attempt to detect reliably the presence of the PU by performing CSS in an ad hoc manner.

\subsection{Channel model and energy-based spectrum sensing}

In this paper, the instantaneous channel power gain from the PU to SUi, $g(i)$, is dependent on the path loss $p(i)$, lognormal shadowing $s(i)$ and multi-path Rayleigh fading $f(i)$ [21]:

$$
g(i)=p(i) \cdot s(i) \cdot f(i)
$$

The path-loss component is given as $p(i)=d_{S P}(i)^{-n}$, where $n$ denotes the path-loss exponent and $d_{S P}(i)$ is the distance between SUi and the PU. The shadowing component in units of decibel (i.e., $10 \log _{10} s(i)$ ) follows a normal distribution with zero mean and standard deviation $\sigma$. The

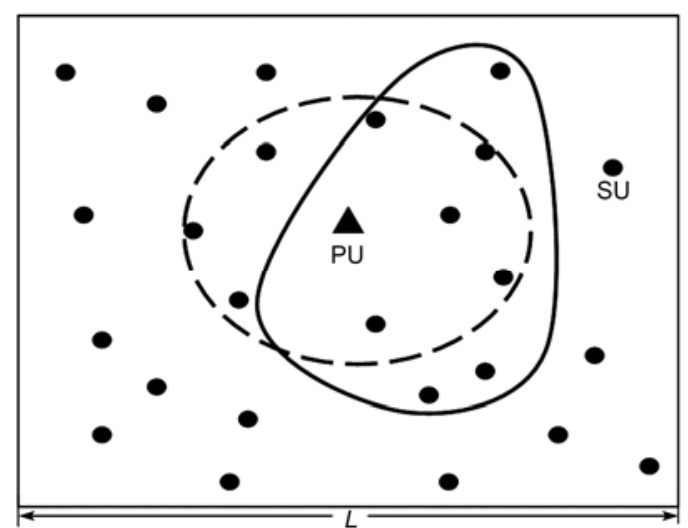

Figure 1 An illustration of the network model. 
multi-path Rayleigh fading $f(i)$ follows an exponential distribution with mean $\mu$. For simplicity, the shadowing and multi-path Rayleigh fading are assumed to be independent for different channels between the PU and SUs.

In the above channel model, each SU locally performs energy-based spectrum sensing [2] to detect the presence of the PU. Let $x(n)$ denote the $n$th signal sample transmitted by the PU and $w(i, n)$ be the $n$th noise sample at SUi. The $n$th sample taken by $\mathrm{SU} i$ can be written as $y(i, n)=h(i) x(n)+$ $w(i, n)$. Here $h(i)=\sqrt{g(i)}$ is the amplitude gain of the channel from the PU to SUi, which is assumed to be slow flat fading during the sampling process. Note that if the PU is absent, $x(n)$ is zero. The total energy collected at SU $i$ is given by $e(i)=\sum_{n=1}^{N_{s}}|y(i, n)|^{2}$. According to the central limit theorem, if the number of collected samples $N_{s}$ is large enough, the sensing result $e(i)$ will be asymptotically normally distributed with mean and variance given by [12]

$$
\begin{gathered}
\boldsymbol{E}[e(i)]=N_{s}[1+\gamma(i)] \delta_{i}^{2}, \\
\operatorname{Var}[e(i)]=2 N_{s}[1+2 \gamma(i)] \delta_{i}^{4},
\end{gathered}
$$

where $\delta_{i}^{2}$ is the variance of noise and

$$
\gamma(i)=1 / N_{s} \sum_{n=1}^{N_{s}}|x(n) h(i)|^{2} / \delta_{i}^{2}
$$

is the average received signal-to-noise ratio (SNR) at $\mathrm{SU} i$.

\subsection{Statement of the problem}

We consider the problem of CSS in a large-scale CRN; i.e., both the dimension of the spatial domain $L$ and the number of SUs $N$ are large. In this case, the sensing overheads and energy consumption are likely to be intolerable if all $N$ SUs participate in CSS. Furthermore, different SUs may have distinctively different detection reliability because of their differing average received SNRs. Intuitively, the SUs (see Figure 1, the nodes in the dashed circle) near the PU possibly have better detection reliability than the SUs far from the PU. However, if we take the shadowing effect and multipath fading into account, the case may not be as simple as that.

On the basis of the above considerations, the goal of this paper is to design a fully decentralized CSS algorithm for the SUs with the best detection performance (see Figure 1, the nodes in the solid circle) instead of all SUs. The first challenge is how to find the potentially best SUs. In traditional centralized CSS, the challenge is usually modeled as a problem of selecting the optimal spectrum sensor set [18], which turns out to be a well-known combinatorial optimization problem with NP-hard complexity. Furthermore, the lack of a central coordinator makes the challenge much more difficult. Other challenges ahead include how to design a proper protocol to summon the potential best SUs and carry out distributed decision-making.

\section{Decentralized CSS in CRNs}

To tackle the above challenges, we propose a consensusbased fully decentralized CSS scheme, which mainly consists of three phases: the clustering phase, the sensing phase and the broadcasting phase. As shown in Figure 2, in the clustering phase, the network attempts to find the SUs with potentially best detection performance in an ad hoc manner; in the sensing phase, the best SUs are summoned for performance decentralized CSS; in the broadcasting phase, the sensing outcomes are broadcast to all other SUs. Let $T_{f}$ denote the total duration of a basic frame consisting of a sensing duration, a broadcast duration and a transmission duration. Note that the clustering phase is activated every $N_{f}=$ $T_{c} / T_{f}$ frames, where $N_{f}$ is a parameter mainly related to the variation in the network topology (e.g., that resulting from the mobility of SUs). Note that the focus of this paper is the design of the clustering phase and the sensing phase. The design of $N_{f}$ is outside the scope of this paper. In the following subsections, we explain the proposed scheme in detail.

\subsection{Phase 1: Consensus-based decentralized clustering}

In this subsection, we "select" the SUs of the best detection performance by tailoring recent advances in unsupervised learning technology [20] and consensus theory [17] to the problem of decentralized CSS. The term "select" in double quotation marks means that there is no central entity taking charge of the selection and each SU makes its own decision (i.e., to join or not to join) on the basis of interactions with one-hop neighbors only.

Let us assume that the SUs are static or quasi-static with slow mobility. In this case, each SU $i$ can obtain an observation $o(i)$ by accumulating its successive $M$ sensing results:

$$
o(i)=\sum_{m=1}^{M} e(i, m),
$$

where $e(i, m)$ is a sensing result; i.e., the collected energy (see Section 2.2) during the $m$ th sensing process. Note that there is no need to spend extra time obtaining the observation $o(i)$. In fact, it can be learned off-line by exploiting historical sensing results. Moreover, the difference in observations among SUs reflects the relative received signal strength or average received SNR when $M$ is large enough.

On the basis of observations $o(i), i \in V$, we introduce a distributed clustering scheme to "select" the SUs having the best detection reliability. Let $K$ denote the number of clusters, $a^{p}(i, k) \in[0,1]$ be the cluster membership coefficient

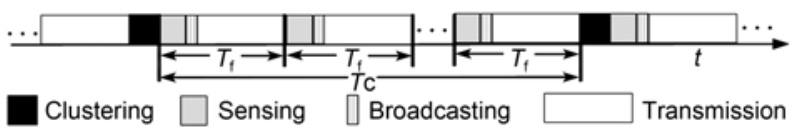

Figure 2 Frame structure of the proposed scheme. 
of $o(i)$ for cluster $k$ and $c(i, k)$ represent the local centroid of cluster $k$ for SUi. By iteratively updating the cluster membership coefficients $\left\{a^{p}(i, k)\right\}$, the local centroids $\{c(i, k)\}$ and the temporary variables $\{w(j, i, k)\}$, the objective of the clustering problem is to determine the optimal membership coefficients by minimizing the sum of squared differences between the observations and the local centroids subject to constraints:

$$
\begin{aligned}
\left\{a^{p^{*}}(i, k)\right\}=\underset{\substack{\left\{a^{p}(i, k)\right\},\{c(i, k)\} \\
\{w(j, i, k)\}}}{\arg \min } \frac{1}{2} \sum_{i=1}^{N} \sum_{k=1}^{K} a^{p}(i, k)\|o(i)-c(i, k)\|^{2},(5) & \begin{array}{l}
\text { sure that all nodes consent to the centroids eventually. To } \\
\text { solve the above optimization problem, we first write the } \\
\text { augmented Lagrangian as }
\end{array} \\
& L\left(\left\{a^{p}(i, k)\right\},\{c(i, k)\}\left\{\lambda_{1}(i, k)\right\},\left\{\lambda_{2}(i, k)\right\}\right) \\
= & \frac{1}{2} \sum_{i=1}^{N} \sum_{k=1}^{K} a^{p}(i, k)\|o(i)-c(i, k)\|^{2}+\sum_{i=1}^{N} \sum_{k=1}^{K} \sum_{j \in N_{i}}\left[\lambda_{1}(i, k)(c(j, k)-w(j, i, k))+\lambda_{2}(i, k)(w(j, i, k)-c(i, k))\right] \\
& +\frac{\eta}{2} \sum_{i=1}^{N} \sum_{k=1}^{K} \sum_{j \in N_{i}}\left(\|c(j, k)-w(j, i, k)\|^{2}+\|w(j, i, k)-c(i, k)\|^{2}\right),
\end{aligned}
$$

$$
\begin{gathered}
\text { s.t. } a^{p}(i, k) \in[0,1], \forall i \in V, \forall k \in\{1, \ldots, K\}, \\
\sum_{k=1}^{K} a^{p}(i, k)=1, \forall i \in V, \\
c(j, k)=w(j, i, k),\{w(j, i, k)\}=c(i, k), \\
\forall i \in V, \forall j \in N_{i}, \forall k \in\{1, \ldots, K\} .
\end{gathered}
$$

Note that the consensus constraints (8) are employed to en- where $\lambda_{1}(i, k)$ and $\lambda_{2}(i, k)$ denote Lagrange multipliers and $\eta$ is an adjustable positive scalar.

The dual optimization problem can be further written as

$$
\begin{aligned}
\min _{\left\{\lambda_{1}(i, k)\right\},\left\{\lambda_{2}(i, k)\right\}}\left\{\max _{\left\{a^{p}(i, k)\right\},\{c(i, k)\}} L\left(\left\{a^{p}(i, k)\right\},\{c(i, k)\}\left\{\lambda_{1}(i, k)\right\},\left\{\lambda_{2}(i, k)\right\}\right)\right\} \\
\text { s.t. } \quad a^{p}(i, k) \in[0,1], \forall i \in V, \forall k \in\{1, \ldots, K\} \\
\quad \sum_{k=1}^{K} a^{p}(i, k)=1, \forall i \in V .
\end{aligned}
$$

According to [20], the solution to eq. (10) can be approximately decomposed into three sequentially iterative subproblems.

(i) Sub-problem 1: Update cluster membership coefficients

$$
\begin{aligned}
a^{p}(i, k)= & \underset{\left\{a^{p}(i, k)\right\}}{\arg \min } \frac{1}{2} \sum_{i=1}^{N} \sum_{k=1}^{K} a^{p}(i, k)\|o(i)-c(i, k)\|^{2} \\
= & \frac{\|o(i)-c(i, k)\|^{-2 /(p-1)}}{\sum_{k=1}^{K}\|o(i)-c(i, k)\|^{-2 /(p-1)}}, \\
& \forall i \in V, \forall j \in N_{i}, \forall k \in\{1, \ldots, K\} .
\end{aligned}
$$

(ii) Sub-problem 2: Update local centroids

$$
\begin{aligned}
c(i, k)= & \left(a^{p}(i, k)+2 \eta\left|N_{i}\right|\right)^{-1} \\
& {\left[a^{p}(i, k) o(i)-2 \lambda(i, k)+\eta \sum_{j \in N_{i}}(c(j, k)+c(i, k))\right], } \\
& \forall i \in V, \forall j \in N_{i}, \forall k \in\{1, \ldots, K\} .
\end{aligned}
$$

(iii) Sub-problem 3: Update local aggregate Lagrange multipliers

$$
\begin{aligned}
\lambda(i, k) & =\lambda(i, k) \\
+ & \frac{\eta}{2} \sum_{j \in N_{i}}(c(j, k)-c(i, k)), \forall i \in V, \forall j \in N_{i}, \forall k \in\{1, \ldots, K\},
\end{aligned}
$$

where $\lambda(i, k)$ denotes a local aggregate Lagrange multiplier. Generally, the obtained cluster membership coefficient $a^{p^{*}}(i, k)$ is a fraction between 0 and 1 . To accomplish the clustering process, we define a hard-decision rule as

$$
a^{p^{*}}(i, k)=\left\{\begin{array}{lr}
1, & k=\arg \max _{k \in\{1, \ldots, K\}} a^{p^{*}}(i, k) \\
0, & \text { otherwise }
\end{array} .\right.
$$

$\mathrm{SU} i \in V_{\text {best }}^{\prime}:=\left\{i \mid a^{p^{*}}\left(i, k^{*}\right)=1, k^{*}=\arg \max _{k} c^{*}(i, k)\right\}$ is then "selected" as the SU with the potentially best detection performance, where $c^{*}(i, k)=c^{*}(j, k), \forall i, j$ is the centroid when convergence of the clustering process is reached.

\subsection{Phase 2: Consensus-based decentralized CSS}

In this subsection, we employ average consensus theory [17] to perform decentralized CSS among the "selected" SUs in $V_{\text {best }}$ with the best detection reliability.

For brevity, we simply cite a theorem concerning the key property of average consensus theory and refer to [17] for details.

Theorem 1 (see [17]): Consider a network of users and let $x_{i}(t)$ denote the consensus variable of user $i$ at time $t$ and the iterative form of the average consensus protocol be

$$
x_{i}(t+1)=x_{i}(t)+\varepsilon \sum_{j \in N_{i}}\left[x_{j}(t)-x_{i}(t)\right]
$$


where $0<\varepsilon<\left\{\max _{i}\left|N_{i}\right|\right\}:=1 / \Delta$ is the step size.

One of the key results is: An average consensus is asymptotically reached with the limit $x^{*}=(1 / N) \sum_{i=1}^{N} x_{i}(0)$ if the network is connected.

As mentioned in Section 1, an average consensus-based decentralized CSS scheme among all SUs has been proposed [16]. However, owing to the collected energies (i.e., $\left.x_{i}(0)=e(i)\right)$ being directly exchanged among one-hop neighbors, that scheme can be regarded as a decentralized implementation of the EGC scheme [12]. Once consensus is reached, each SU locally obtains the final decision by comparing the consensus variable value $x^{*}$ with a predefined threshold $\lambda$ :

$$
D=\left\{\begin{array}{l}
1, \quad x^{*}>\lambda, \\
0, \text { otherwise }
\end{array}\right.
$$

Similarly, if we redefine the initial consensus variables as

$$
x_{i}(0)=\frac{\gamma(i)}{1+\gamma(i)} e(i), \forall i
$$

we then obtain a decentralized implementation of the optimal soft combination (OSC) scheme [12], which usually outperforms the EGC scheme. Note that the priori estimation of the local SNR $\gamma(i), \forall i \in V_{\text {best }}$ is needed for each SU in the OSC scheme.

To reduce the complexity and sensing overheads, we declare that: After the phase of decentralized clustering, only the "selected" SUi, $\forall i \in V_{\text {best }}$ with the potentially best detection reliability participates in decentralized CSS according to the average consensus protocol [15] and the initial consenus variable is $x_{i}(0)=\mathrm{e}(i)$. Note that the one-hop neighbor set $N_{i}$ of SUi, $\forall i \in V_{\text {best }}$ has been updated to $N_{i, \text { best }}:=\left\{j \mid j \in V_{\text {best }}, \boldsymbol{A}_{i j}=1\right\}$.

Finally, in Phase 3, the "selected" SUs in $V_{\text {best }}$ broadcast the sensing outcomes to the other SUs outside the cluster.

\subsection{The proposed decentralized CSS scheme}

In this subsection, we summarize of the proposed scheme.

Algorithm 1: Consensus-Based Decentralized Clustering for Cooperative Spectrum Sensing

Initialization: According to eq. (4), each SUi obtains its observation $o(i)$ by off-line learning.

Phase 1: Consensus-based decentralized clustering

$$
\begin{aligned}
& \text { for } t=1,2 \ldots \text { do } \\
& \quad \text { for } i=1, \ldots, N \\
& \quad \text { for } k=1, \ldots, K
\end{aligned}
$$

Calculate the membership coefficient $a^{p}(i, k)$ according to eq. (11);

Calculate the local centroids $c(i, k)$ of cluster $k$ for SU $i$ according to eq. (12);

Update the local aggregate Lagrange multiplier $\lambda(i, k)$ according to eq. (13).

$$
\text { End }
$$

End

End

Each SUi makes its local decision according to eq. (14).

Phase 2: Consensus-based decentralized CSS

Each SU $i$ in $V_{\text {best }}$ performs local sensing and sets its initial consensus variable $x_{i}(0)=e(i)$.

for $t=1,2 \ldots$ do

Each SUi in $V_{\text {best }}$ exchanges its consensus variable $x_{i}(t)$ with its one-hop neighbors according to the protocol given by eq. (15).

End

Phase 3: Broadcasting

The SUs in $V_{\text {best }}$ broadcast the sensing outcomes to other SUs outside the cluster.

\section{Performance evaluation and discussion}

\subsection{Simulation setup}

In this section, we compare the detection performance of the proposed scheme with the performances of existing schemes. The bandwidth $W$ is $10 \mathrm{MHz}$ and the sensing time is $100 \mu \mathrm{s}$. The noise spectral density is $N_{0}=-174 \mathrm{dBm}$. As shown in Figure 3(a), we consider an $10 \mathrm{~km} \times 10 \mathrm{~km}$ area with a PU located at the center. There are 36 SUs uniformly distributed in the area. The transmission power of the PU is $200 \mathrm{~mW}$. The path-loss exponent is 4 , the standard deviation of the shadow is $10 \mathrm{~dB}$ and the mean of the multi-path Rayleigh fading is assumed to be 1 .

\subsection{Results for different clustering schemes}

Figure 3(b) presents the results for the proposed consensusbased decentralized clustering scheme, where seven SUs are "selected" as the SUs with potentially best detection performance. For comparison, we also give the results for the location-awareness (or distance-based) decentralized clustering scheme (see Figure 3(c)), in which the seven SUs nearest the PU are "selected". The difference between Figure 3(b) and (c) results from the fact that the former has taken the integrated effects of path loss, shadowing and multi-path fading into account while the later considers the path-loss effect only.

\subsection{Comparison of the receiver operating characteris- tic (ROC) curves}

In Figure 4, we compare the ROC curves of the proposed scheme, the OSC scheme [12], the EGC scheme [16], and the location-awareness clustering scheme. The figure shows that the proposed scheme significantly outperforms the EGC scheme and the location awareness scheme, and achieves detection performance comparable to that of the OSC scheme. 

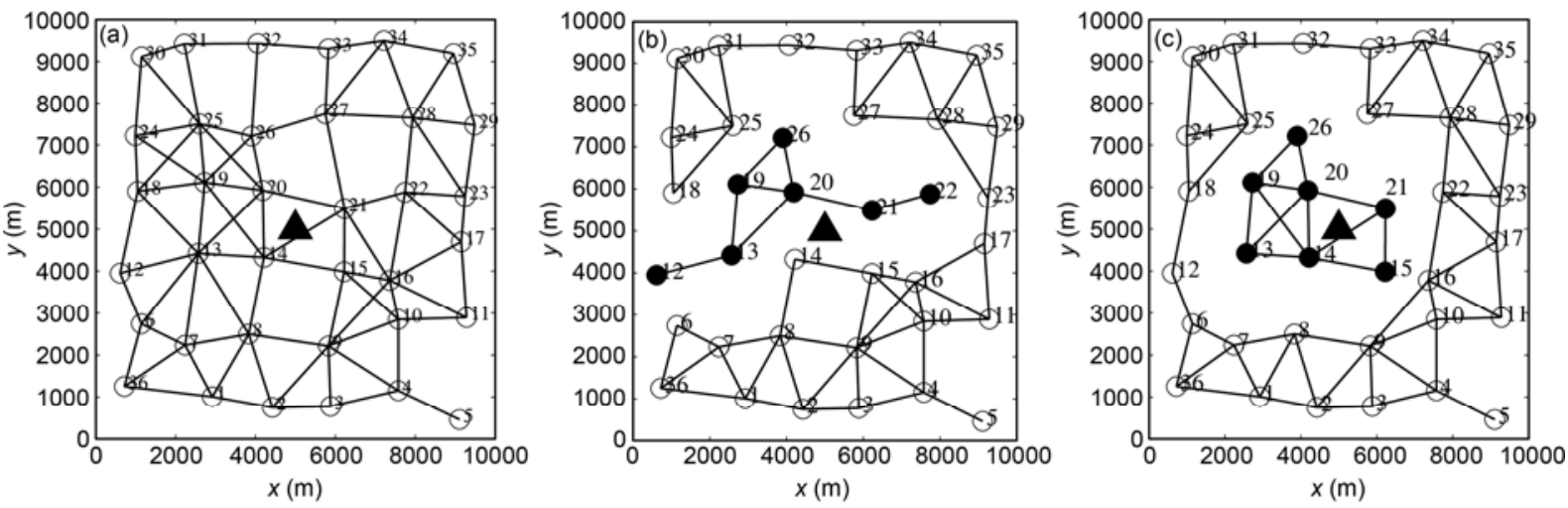

Figure 3 Network used for simulation and the results for different clustering schemes; the triangle denotes the PU, the solid circles represent the "selected" SUs and the open circles are SUs that do not participate in CSS. (a) The network used for simulation; (b) the proposed clustering scheme; (c) the location-awareness clustering scheme.

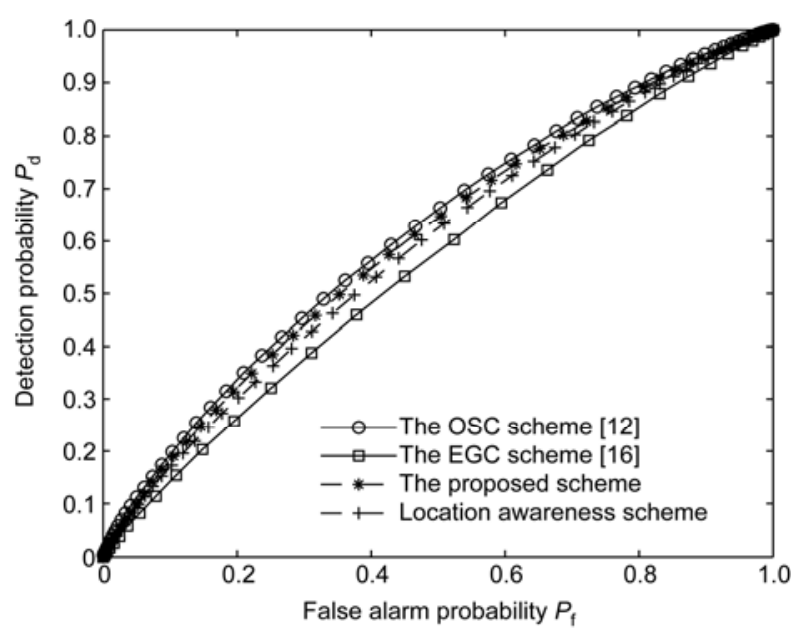

Figure 4 Comparison of the ROC curves.
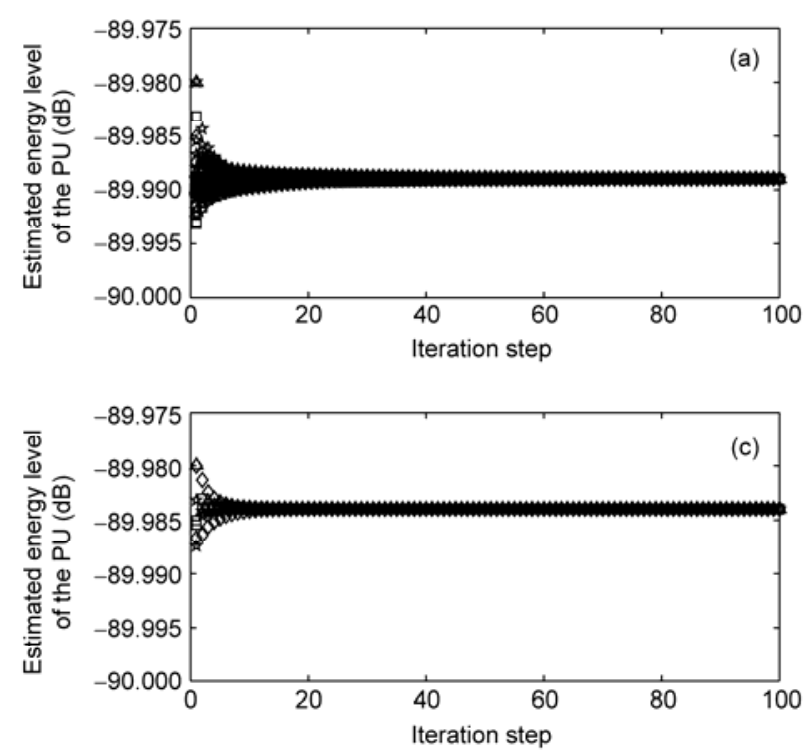

\subsection{Comparison of the convergence performance}

In Figure 5, we compare the convergence performance of different schemes. We can see that compared with the decentralized EGC scheme [16] and the decentralized OSC scheme [12], where all SUs participate in CSS, the proposed scheme has a much higher convergence speed, which means a much reduced sensing time and much lower energy consumption. We also see that the location-awareness scheme has convergence performance similar to that of the proposed scheme. However, the proposed scheme does not need to estimate the distance between the SUs and the PU, which can significantly reduce the system complexity.

\subsection{Complexity of different schemes}

In Table 1, we compare the complexity of different schemes
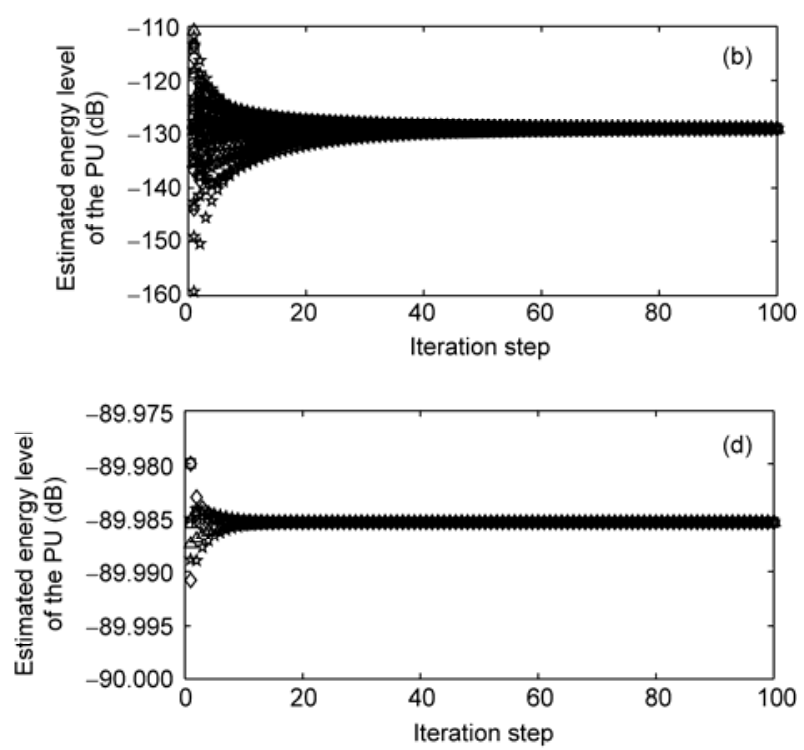

Figure 5 Comparison of the convergence performances of different schemes; the $x$-axis denotes the iteration time and the $y$-axis denotes the evolution of the estimated energy level of the PU for different SUs participating in CSS. (a) Decentralized EGC [16]; (b) decentralized OSC [12]; (c) the proposed scheme; (d) location-awareness scheme. 
Table 1 Comparison of the complexity of different schemes

\begin{tabular}{lcccc}
\hline & Decentralized EGC [16] & Decentralized OSC [12] & Location-awareness & The proposed \\
\hline SUs involved in CSS & All & All & Partial & Partial \\
Location estimation & No & No & Yes & No \\
SNR estimation & No & Yes & No & No \\
\hline
\end{tabular}

in terms of the number of SUs involved in CSS, location estimation of the PU, and estimation of the received SNR. The advantages of the proposed scheme include that only some SUs are involved in CSS and no location or SNR estimation is needed. Note that compared with traditional EGC and OSC schemes, the clustering process of the proposed scheme introduces additional complexity; however, if the network topology is fixed, which is the most common hypothesis in the literature (see [16] and [12], among others), the clustering process should be activated only in the initialization phase of the network.

\section{Summary and future directions}

A fully decentralized cooperative spectrum sensing scheme is proposed on the basis of recent advances in unsupervised learning technology and consensus theory. The proposed scheme mainly consists of two phases-the clustering phase and sensing phase-both of which are implemented in an ad hoc manner. The clustering phase aims at clustering the SUs with potentially best detection performance and the sensing phase carries out distributed data fusion of the sensing outcomes of the SUs in the cluster. Numerical results show that the proposed scheme achieves detection performance comparable to that of the OCS scheme with significantly reduced sensing overheads. A future direction of study is to consider the quantized effect of the sensing outcomes on the detection and convergence performance. Another direction is to analyze sensing overheads quantitatively.

This work was supported by the National Basic Research Program of China (2009CB320400), the National Natural Science Foundation of China (60932002 and 61172062), and the Natural Science Foundation of Jiangsu, China (BK2011116).

1 Tandra R, Mishra S M, Sahai A. What is a spectrum hole and what does it take to recognize one. Proc IEEE, 2009, 97: 824-848

2 Ma J, Li G, Juang B H. Signal processing in cognitive radio. Proc IEEE, 2009, 97: 805-823

3 Cabric D, Mishra S M, Brodersen R. Implementation issues in spectrum sensing for cognitive radios. In: Michael B M, ed. Proceedings of 38th Asilomar Conference on Signals, Systems, and Computers. Pacific Grove, USA, 2004. 772-776

4 Sahai A, Tandra R, Mishra S M, et al. Fundamental design tradeoffs in cognitive radio systems. In: Milind $\mathrm{B}$, Anant $\mathrm{S}$, Kitti $\mathrm{H}$, eds.
Proceedings of the First International Workshop on Technology and Policy for Accessing Spectrum. Boston, USA, 2006. 1-6

5 Duan D L, Yang L Q, Principe J C. Cooperative diversity of spectrum sensing for cognitive radio systems. IEEE Trans Signal Process, 2010, 58: 3218-3227

6 Yucek T, Arslan H. A survey of spectrum sensing algorithms for cognitive radio applications. IEEE Commun Surveys Tuts, 2009, 11: $116-130$

7 Zeng Y H, Liang Y C, Hoang A T, et al. A review on spectrum sensing for cognitive radio: Challenges and solutions. EURASIP J Adv Signal Process, 2010: 1-15, doi:10.1155/2010/381465

8 Shen J Y, Jiang T, Liu S Y, et al. Maximum channel throughput via cooperative spectrum sensing in cognitive radio networks. IEEE Trans Wireless Commun, 2009, 8: 5166-5175

9 Jayakrishnan U, Venugopal V V. Cooperative sensing for primary detection in cognitive radio. IEEE J Sel Topics Signal Process, 2008, 2: $18-27$

10 Peh E C, Liang Y C, Guan Y L, et al. Optimization of cooperative sensing in cognitive radio networks: A sensing-throughput tradeoff view. IEEE Trans Veh Technol, 2009, 58: 5294-5299

11 Quan Z, Ma W, Cui S G, et al. Optimal linear fusion for distributed detection via semi-definite programming. IEEE Trans Signal Process, 2010, 58: 2431-2436

12 Ma J, Zhao G, Li Y. Soft combination and detection for cooperative spectrum sensing in cognitive radio networks. IEEE Trans Wireless Commun, 2008, 7: 4502-4507

13 Miia M, Marja M, Aarne M. Cooperative spectrum sensing using quantized soft decision combining. In: Souhir D, Joao P M, eds. Proceedings of 4th International Conference on Cognitive Radio Oriented Wireless Networks and Communications. Hannover, Germany, 2009. 1-5

14 Wang B B, Liu K J R, Clancy T C. Evolutionary cooperative spectrum sensing game: How to collaborate? IEEE Trans Commun, 2010, 58: 890-900

15 Saad W, Han Z, Debbah M, et al. Coalitional games for distributed collaborative spectrum sensing in cognitive radio networks. In: Artur $\mathrm{Z}$, Li Q, eds. Proceedings of 28th Conference on Computer Communications. Rio de Janeiro, Brazil, 2009. 2114-2122

16 Li Z Q, Yu F R, Huang M Y. A distributed consensus-based cooperative spectrum sensing scheme in cognitive radios. IEEE Trans Veh Technol, 2010, 59: 383-393

17 Reza O S, Fax J A, Richard M M. Consensus and cooperation in networked multi-agent systems. Proc IEEE, 2007, 95: 215-233

18 Zaheer K, Janne L, Kenta U, et al. On the selection of the best detection performance sensors for cognitive radio networks. IEEE Signal Process Lett, 2010, 17: 359-362

19 Guo C, Peng T, Xu S Y, et al. Cooperative spectrum sensing with cluster-based architecture in cognitive radio networks. In: Guillem F, ed. Proceeding of 69-th Vehicular Technology Conference: VTC2009Spring. Bacolona, Spain, 2009. 1-5

20 Pedro A F, Alfonso C, Georgios B G. Distributed clustering using wireless sensor networks. IEEE J Sel Topics Signal Process, 2011, 5: 707-724

21 Andrea G. Wireless Communications. London: Cambridge University Press, 2005

Open Access This article is distributed under the terms of the Creative Commons Attribution License which permits any use, distribution, and reproduction in any medium, provided the original author(s) and source are credited. 